\title{
Can respiratory physiology predict thermal niches?
}

\author{
Wilco C.E.P. Verberk, ${ }^{1}$ Fabrizio Bartolini, ${ }^{2}$ David J. Marshall, ${ }^{3}$ Hans-O. Pörtner, ${ }^{4}$ \\ John S. Terblanche, ${ }^{5}$ Craig R. White, ${ }^{6}$ and Folco Giomi ${ }^{4}$ \\ ${ }^{1}$ Department of Animal Ecology and Ecophysiology, Radboud University Nijmegen, Nijmegen, the Netherlands. ${ }^{2}$ Université \\ Nice Sophia Antipolis, ECOMERS, Nice, France. ${ }^{3}$ Faculty of Science, Universiti Brunei Darussalam, Brunei. ${ }^{4}$ Department of \\ Integrative Ecophysiology, Alfred-Wegener-Institute for Polar and Marine Research, Bremerhaven, Germany. ${ }^{5}$ Department of \\ Conservation Ecology and Entomology, Centre for Invasion Biology, Stellenbosch University, Stellenbosch, South Africa. \\ ${ }^{6}$ School of Biological Sciences, The University of Queensland, Brisbane, QLD, Australia
}

Address for correspondence: Wilco C.E.P. Verberk, Department of Animal Ecology and Ecophysiology, Radboud University Nijmegen, Nijmegen, the Netherlands. wilco@aquaticecology.nl

Predicting species responses to global warming is the holy grail of climate change science. As temperature directly affects physiological rates, it is clear that a mechanistic understanding of species vulnerability should be grounded in organismal physiology. Here, we review what respiratory physiology can offer the field of thermal ecology, showcasing different perspectives on how respiratory physiology can help explain thermal niches. In water, maintaining adequate oxygen delivery to fuel the higher metabolic rates under warming conditions can become the weakest link, setting thermal tolerance limits. This has repercussions for growth and scaling of metabolic rate. On land, water loss is more likely to become problematic as long as $\mathrm{O}_{2}$ delivery and $\mathrm{pH}$ balance can be maintained, potentially constraining species in their normal activity. Therefore, high temperatures need not be lethal, but can still affect the energy intake of an animal, with concomitant consequences for long-term fitness. While respiratory challenges and adaptive responses are diverse, there are clear recurring elements such as oxygen uptake, $\mathrm{CO}_{2}$ excretion, and water homeostasis. We show that respiratory physiology has much to offer the field of thermal ecology and call for an integrative, multivariate view incorporating respiratory challenges, thermal responses, and energetic consequences. Fruitful areas for future research are highlighted.

Keywords: climate change; ectotherms; metabolic rates; oxygen limitation; thermal biology; water balance

\section{Introduction}

Respiration is a key feature of animal life, usually with oxygen being used by the mitochondria in oxidative phosphorylation as the prime mechanism that provides metabolic energy. At the same time, temperature is a key environmental driver of animal life, since it determines the energetic costs for maintenance and the rates at which energy can be gained. Considering that - all else being equal — the energy available to an organism represents a useful measure of potential fitness, it is clear that the interplay between respiratory physiology and thermal biology has much to offer in understanding the ecology and evolution of species. Here, we focus on these aspects to point out the links between respiratory functions and thermal niches of species. Temperature directly affects metabolism in ectotherms and hence their respiration. For endotherms, temperature modulates the allocation of energy to either heat generation or dissipation, which alters oxygen requirements. What about the reverse: what can respiration physiology tell us about how animals can cope with extreme or variable temperatures (i.e., their thermal niche)? Here, we offer different viewpoints on how respiratory physiology may contribute to understanding species thermal adaptation, with topics exemplified by terrestrial insects, intertidal snails, and aquatic eurytherms. The review is not an attempt to be complete, but rather aims to highlight key developments that showcase how respiratory physiology shapes the thermal niche of a species and to propose promising directions for future research to better understand and forecast species responses to climate warming. 


\section{Metabolic scaling and the thermal niche}

Temperature has a pervasive effect on the physiological rates of animals (e.g., Ref. 1), including a species' energy metabolism, which is closely approximated by the rate at which it consumes oxygen. Therefore, intuitively, the metabolic and thermal aspects of a species' niche should be tightly connected to its respiratory physiology. However, explaining a species' performance from its metabolic rate is less than straightforward. This could be related to the variability inherent in metabolism. Indeed, metabolic rate can be viewed both as a boon and a burden, either representing the flow of energy and hence the capacity of an organism to be active, grow, and reproduce (aerobic scope) or representing the energetic maintenance costs (standard metabolic rate).

A major source of variation in metabolism is linked to the body size of the organism. Not surprisingly, larger animals require more oxygen. What is surprising, however, is the observation that metabolic rate does not increase in direct proportion to body size (e.g., Refs. 2-4). Instead, the relationship is allometric, such that metabolic rate (MR) scales with body mass $(M)$ approximately as a power function (MR $\propto M^{b}$, where $b$ is a scaling exponent). The value of this exponent is usually smaller than 1 , indicating that larger animals consume less oxygen relative to their body size, which may indicate that there are advantages associated with being large. While early work generally supported the hypothesis that metabolic rate scaled in proportion to body surface area (e.g., $b=2 / 3$ ), ${ }^{2,5}$ later the exponent $b=3 / 4$ came into widespread use. ${ }^{6}$

The relationship between body mass and metabolism continues to be an active area of research (for reviews, see Refs. 7 and 8). Elucidating the exact value of the scaling component has farreaching consequences for ecology, given the central importance of scaling relationships in many models, including those for ontogenetic growth, a key component of the ecological niche. ${ }^{9,10}$ Despite a broad consensus by the 1980s that metabolic rate scaled, on average, with an exponent of $3 / 4$, there has long been evidence against the universality of any single value. ${ }^{9,11,12}$ The scaling exponent of metabolic rate has, for example, been shown to vary between endotherms and ectotherms, ${ }^{13,14}$ among taxonomic groups, ${ }^{15,16}$ among activity states, ${ }^{15,17}$ and along the gradient in body size itself. $^{16,18}$
While linking metabolism directly to ecological fitness has proven difficult, evidence of associations between the scaling of metabolic rates of species and characteristics of their ecological niches are beginning to accumulate. This suggests not only that the value of the metabolic scaling exponent is variable, but that the observed variation has ecological causes or consequences. There are a number of examples for both biotic (intrinsic, biological) and abiotic (extrinsic, environmental) effects on the scaling exponent of metabolic rate (reviewed in Refs. 11 and 19). For example, the scaling exponent of metabolic rate is lower in benthic (bottom-dwelling) aquatic animals than in pelagic (open-water) ones, ${ }^{20}$ and amphipods such as Gammarus minus that live with predators have a lower metabolic scaling exponent than those that do not. ${ }^{21}$ Examples of extrinsic, abiotic influences on the scaling exponent of metabolic rate often pertain to resource availability. For example, decreases in the scaling exponent were found for light limitation in phytoplankton, ${ }^{22}$ dietary imbalance in Daphnia, ${ }^{23}$ and food restriction in Manduca sexta caterpillars, albeit not significantly. ${ }^{24}$ In contrast, scaling exponents were found to increase due to starvation in shore crabs (Carcinus maenus), ${ }^{25}$ sand dollars (Mellita quinquiesperforata), ${ }^{26}$ and fish. ${ }^{27,28}$ Recent observations suggest that rapid growth and shallow scaling exponents are associated, providing a quantitative link between physiological scaling and mechanistic models for ontogenetic growth. ${ }^{10,29}$

When one turns to the effects of temperature on metabolic scaling, the most consistent effects have been found for fish, and these had shallower scaling exponents of metabolic rate with increasing temperature. ${ }^{30}$ This pattern is consistent with both Glazier's hypothesis that the metabolic level of an animal itself sets boundaries to metabolic scaling ${ }^{7,11}$ and with the recent hypothesis that the challenge of gas exchange underwater is dependent on size. ${ }^{31}$ Breathing underwater is especially challenging for small animals, for whom viscosity effects are more pervasive, and this size difference becomes more pronounced in cold water, which is more viscous ${ }^{31}$ (see also next section). Ivleva ${ }^{32}$ also found a decrease in the scaling exponent with increasing temperature for crustaceans, but support from less comprehensive studies is mixed (see Refs. 7 and 11 for comprehensive reviews). A valuable next step in distinguishing among alternative 
hypotheses for metabolic scaling ${ }^{7,11,31}$ would be to investigate whether the mass scaling exponent for standard metabolism of air-breathing ectotherms is likewise temperature dependent (as predicted by Glazier's hypothesis) or independent (as predicted by the size-dependent viscosity effect of water). Establishing whether increasing temperature consistently causes shallower slopes in both air and water breathers will also help reveal whether rapid growth and shallow scaling exponents are intrinsically linked or not.

\section{Balancing oxygen supply and demand while breathing water or air: regulatory ability as a key trait}

Respiration represents a delicate trade-off, as oxygen is both toxic and essential for aerobic energy generation. Toxic effects of oxygen arise as oxygen radicals are produced in the mitochondria in an oxygen-dependent manner. While toxicity effects are most readily manifested at high partial pressures of oxygen, when cellular defense mechanisms are overwhelmed, even normoxic levels have been shown to induce extensive oxidative damage both in vivo and in vitro, ${ }^{115}$ contributing to the wear and tear that we experience as aging. ${ }^{116}$ This is because, at normoxia, oxygen partial pressure $(21 \mathrm{kPa})$ is still approximately 50 times higher than that at which mitochondria function optimally $(0.3-0.5 \mathrm{kPa})$. As such, it is important for organisms to deliver just the right amount of oxygen to respiring tissues, and the respiratory system can perhaps be usefully characterized as a gatekeeper of oxygen distribution. ${ }^{33}$ Thus, an animal must be able to reduce the risk of oxygen toxicity, while at the same time retaining sufficient scope for oxygen uptake across different levels of activity to avoid asphyxiation. ${ }^{31}$ These risks go hand in hand: while a greater capacity to take up oxygen would do away with asphyxiation risks, such an overdesigned respiratory system would come at the high price of oxygen toxicity. For example, avoiding oxygen toxicity is one of the hypotheses put forth to explain why many terrestrial insects breathe discontinuously when at rest. ${ }^{34}$ The tight balancing act between the risks of oxygen toxicity and asphyxiation may explain why the oxygen requirements of an organism and its capacity to supply oxygen are frequently matched, as recognized by the principle of symmorphosis, ${ }^{35}$ and may have played a role in the recurrent evolution of large body sizes during geological periods when atmospheric oxygen levels were putatively high (Carboniferous and end-Cretaceous). ${ }^{31,117}$

With temperature and body mass constituting two primary influences on metabolic rate, ${ }^{36}$ it is of interest to evaluate how both these factors affect the balancing act of respiration. For this, one needs to differentiate between respiratory media, as respiratory challenges are very different for organisms breathing air or water. ${ }^{31}$ Air contains much more oxygen than water $\left(30\right.$ times more at $\left.20^{\circ} \mathrm{C}\right)$, and, in addition, diffusivity of oxygen is about four orders of magnitude greater in air than in water. ${ }^{37} \mathrm{In}$ water, temperature and body size affect the balance between the oxygen requirements of an organism (oxygen demand) and its capacity to deliver oxygen (oxygen supply) (Fig. 1). Temperature affects both oxygen demand and the bioavailability of oxygen in water, which generally gives rise to a progressive mismatch between oxygen supply and demand with increasing temperatures, ${ }^{38,41}$ since the capacity of oxygen-supply mechanisms is limited. ${ }^{39}$ Body size likewise increases oxygen demand and supply, which again results in a mismatch as larger organisms generally have smaller (respiratory) surface area-to-volume ratios. These generalities beguile a deeper complexity, where effects of temperature on viscosity and effects of body size on the ventilatory movement of water across respiratory surfaces result in differences in ventilatory efficiency. ${ }^{31}$ The ventilation efficiency, in turn, is essential for regulating oxygen uptake, which plays an obvious role in balancing risks of oxygen toxicity and asphyxiation.

Whether gas exchange takes place in air or water, ultimately all organisms have to deal with the lower diffusivity and solubility of oxygen in water. This is because even air breathers will ultimately perform gas exchange across a watery environment (e.g., a thin aqueous film in tetrapod lungs, wet skin in amphibians, and fluid-filled tracheole in insects). What makes breathing under water a challenge is the much larger effort of ventilation required in water compared to air owing to the higher density and viscosity of water, meaning that greater efforts are required to create a sufficiently steep gradient in partial pressure of oxygen to facilitate oxygen diffusion across respiratory surfaces. This also means that the ability of organisms to dynamically change and regulate oxygen uptake (i.e., their regulatory ability) is inherently more limited in water than 


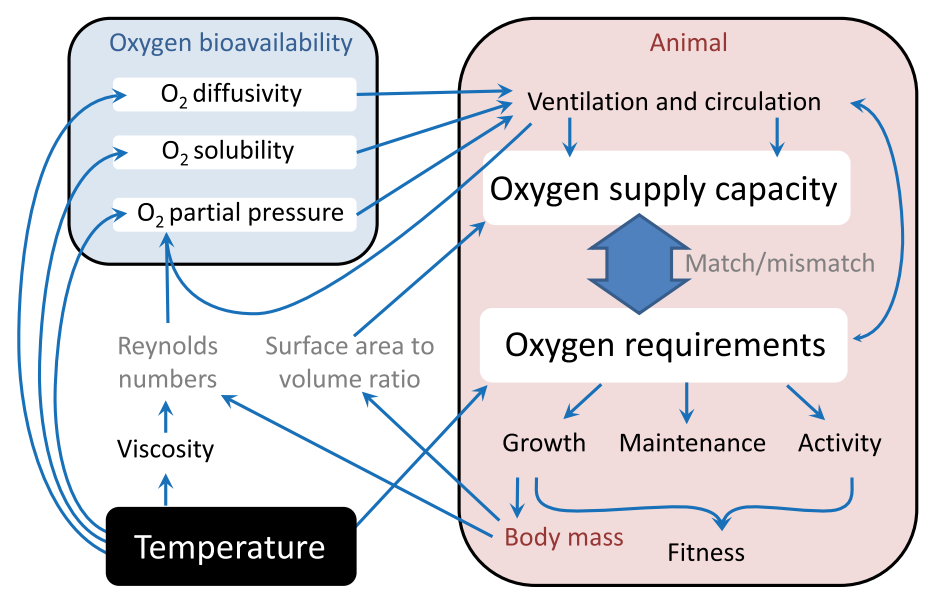

Figure 1. Overview of the myriad effects of temperature, both direct and indirect, on both oxygen-supply capacity and oxygen requirements. Adapted from Refs. 31 and 38.

in air. ${ }^{31}$ One way to reduce the cost of breathing in water is the adoption of cutaneous respiration, given that this requires no ventilatory effort. Indeed, virtually all aquatic ectotherms use cutaneous respiration to augment their oxygen uptake. However, as animals can exert relatively little control on the passive diffusion of oxygen across their integument, a higher reliance on cutaneous respiration only further reduces the capacity of water breathers to regulate oxygen uptake, ${ }^{31}$ explaining the greater sensitivity of skin breathers to fluctuations in temperature and environmental oxygen conditions. ${ }^{40}$

Both temperature and body size affect ventilation efficiency. Oxygen diffusion is reduced in cold water owing to the thermal dependency of diffusivity. In addition, cold water is more viscous than warm water, further impeding oxygen diffusion because a thicker layer of water clings to respiratory surfaces (thicker boundary layers) $^{38,41}$ (Fig. 1). A greater ventilatory effort is needed to disrupt these boundary layers and restore contact between the respiratory surfaces and fresh oxygenated water. Contrasting with these negative effects of cold water on oxygen supply is the greater solubility of oxygen in cold water. Hence, cold water is less likely to become depleted of oxygen. Thus, the need for costly ventilation is also reduced, allowing greater ventilatory efficiency in cold water. ${ }^{31} \mathrm{~A}$ cold-induced reduction in oxygen demand further ameliorates this situation. To complicate matters, the viscosity-related effects are size dependent, affecting small organisms much more strongly than larger organisms (Fig. 1). Thus, the consequences of warming for underwater gas exchange are very context dependent, depending on the oxygen consumption of the organism, its speed and size (which govern how much it is affected by viscosity in the first place), and its rate of ventilation. Small animals, which are heavily affected by viscosity, with low oxygen-consumption rates or high ventilation rates will be more likely to experience increased oxygen availability in warm waters, while large animals with high oxygen consumption and low ventilation will be more likely to face decreased oxygen availability. These size differences in ventilatory ability may help explain the phenomenon that body size is reduced whereas growth rates are enhanced in warmer waters, ${ }^{31,42}$ a phenomenon that seems to be more prominent in aquatic than in terrestrial ectotherms. ${ }^{43}$

The concept of oxygen- and capacity-limited thermal tolerance (OCLTT) envisages a key role for respiratory physiology in limiting thermal performance through the development of a mismatch in whole-organism oxygen supply and demand. The recognition that regulatory ability is far more difficult or energetically costly to achieve in water than in air may help explain differences in the thermal biology of air breathers and water breathers. For example, Verberk and Bilton ${ }^{44,119}$ compared various insects covering a range of respiratory strategies (e.g., air, gill, and plastron breathers), exhibiting contrasting ability to regulate oxygen uptake, and demonstrated that species with poor regulatory abilities (aquatic animals with gill 
and tegument breathing) were consistently more vulnerable to the combined effects of heat and hypoxia. This demonstrates that differences in regulatory ability are linked to the degree to which their heat tolerance is limited by oxygen. In a related vein, Giomi and collegues ${ }^{45}$ linked such improved heat tolerance in air to the recurrent evolution of air-breathing. This finding is further supported by the great species richness of air-breathing crabs and fishes within the tropics, where the high environmental temperatures drive the frequent and independent transition from water-breathing to air-breathing forms. Similarly, the capacity of the tropical eurythermal shrimp Macrobrachium rosenbergii to sustain aerobic metabolism and oxygen delivery even at high temperatures would predict that species to have excellent regulatory ability. ${ }^{46}$ The well-known progressive lowering of the affinity of oxygen-binding proteins with increasing temperatures could explain how eurytherm crustaceans maintain efficient oxygen delivery at high temperatures. This mechanism has been demonstrated for the green crab (Carcinus maenas) ${ }^{47}$ showing that during acute heating the progressive hypoxemia and the resultant hemolymph acidosis greatly facilitated the unloading of oxygen into tissues. Thus, organisms with a good oxyregulatory ability may better tolerate high temperatures. Being able to accurately meet temperature-induced fluctuations in oxygen demand safeguards organismal energy status and performance, which ultimately govern population and ecosystem dynamics.

\section{Respiration of eurytherms and stenotherms: a trade-off between thermal specialization and performance}

The dimensions of a species' thermal niche arise from the concerted effects of genetic traits, epigenetic inheritance, and plastic responses to local environmental thermal conditions. ${ }^{48-52}$ Depending on the stability of thermal conditions, selection has given rise to widening of the thermal niche or to distinct thermal specialization..$^{53,118}$ So-called eurythermal species have a wide thermal niche, occupying contrasting and highly variable thermal environments. Eurythermal species display a great functional capacity across a wide range of thermal conditions, thanks to their large scope for phenotypic plasticity acting on different hierarchical levels. ${ }^{48,54}$ Among others, this includes the temperature-responsive expression of different isoforms of genes and temperature-dependent adjustments of mitochondrial function and of the capacity of compensatory mechanisms of systemic processes, such as the tuning of respiratory, cardiovascular, and ventilatory systems. ${ }^{51,55,56}$ Conversely, the adaptive processes driven by stable climatic conditions and limited temperature fluctuation (seasonal and daily) operate toward thermal specialization, setting narrower thermal performance windows and promoting the evolution of stenothermal forms. ${ }^{48,57,58} \mathrm{~A}$ driving force for the evolution of thermal specialization relates to the energy savings involved in a stenothermal mode of existence. ${ }^{59}$

The coexistence of thermal generalists and specialists suggests that a trade-off exists between the level of performance at the thermal optimum and the breadth of the thermal niche, which in turn gives rise to stenothermal species that can successfully outcompete their eurythermal counterparts, but only under stable environmental conditions, and eurythermal species that are more successful in more variable environments. ${ }^{48}$ Evaluating such a trade-off, however, requires knowledge of the underlying physiological mechanisms. Temperature-induced mismatches between oxygen-delivery capacity and oxygen demand and the heat thresholds that bring a species to hypoxemic conditions have been suggested to underlie thermal tolerance limits in ectotherm species. ${ }^{51}$ Consequently, thermal specialists could be especially limited by their capacity to increase oxygen uptake to meet demand in warm conditions. ${ }^{44,45,119}$

Recent attempts to predict species' vulnerability and resilience to climate change rely heavily on their presumed respiratory physiology and the differences therein between thermal generalist and specialist species. ${ }^{60,61}$ With the emphasis on acute heat stress, it has been proposed that the actual trend of global warming would provide generalist eurytherms with a clear advantage compared to specialist stenotherms. ${ }^{62-65}$ These studies estimate absolute changes of ectotherm metabolic rates at a global scale and envisage that tropical specialists living close to the upper limit of their thermal niche could experience the largest increases in metabolic demand. If their capacity to meet demand is thermally impaired, this would result in a rapid fall-off of observed metabolic rates at critical temperatures. Indeed, in tropical stenotherms, owing to a narrow 
breadth of the thermal niche and the steep slope of the metabolic curve, minor environmental warming would lead to stronger increases in metabolic costs than in eurytherm species. Validations or refutations of these projections critically depend on both how eurytherms and stenotherms can tune their metabolic plasticity and how they can adapt to climate warming.

With regard to metabolic plasticity, experimental studies suggest that thermal specialists may potentially face anomalous climatic events more effectively than their generalist counterparts, contrasting with the predictions above. ${ }^{66}$ These studies demonstrate how thermal specialists may acclimatize and efficiently shift their thermal-tolerance windows on a seasonal basis. Such seasonal acclimation of metabolic costs indicates the potentially adaptive response of thermal specialists and their capacity to optimize energy demand in response to temperature variations. ${ }^{51}$ Seasonal acclimatization involves different components of respiratory function (e.g., ventilation activity, oxygen consumption, affinity of oxygen-binding proteins) and shows that the thermal optimum for respiratory performance shifts toward higher temperatures when animals move from cold to warm seasons. ${ }^{67}$

Acclimatization potential has also recently been studied by comparing the respiratory functions of populations at different latitudes, and the results provide empirical support for the notion that thermal specialization may be adaptive at tropical latitudes. Specifically, Fusi et al. ${ }^{68}$ have shown that eurythermal mangrove crabs are able to withstand thermal conditions fluctuating on a diurnal and seasonal basis, sustaining functional capacity across a wide range of temperatures. However, while these populations are adapted to a wide thermal range, they are vulnerable to extreme heat events that extend beyond their range of environmental temperatures, showing a weak capacity to endure anomalous climate warming. In contrast, tropical stenotherms were able to endure such anomalous heat stress. They maintained functional capacity by increasing respiratory function to effectively meet the heat-induced increase in oxygen demand (Fig. 2).

It seems that stenotherms rely more heavily on their acclimation potential than eurytherms to withstand cyclical or anomalous temperature fluctuations. It is possible that eurytherms need to resort less to acclimatory responses to deal with temperature changes owing to their broad thermal window. Taken together, this suggests that stenotherms may have a larger scope for acclimatization than eurytherms. The explanation for a larger scope for acclimatization in stenotherms may be rooted in the physiological costs that drive the eurytherm-stenotherm trade-off. The evolution of thermal niches of increasing width is paralleled by the rising severity of environmental stress that organisms undergo and whose tolerance is directly limited by bioenergetic constraints. ${ }^{69}$ To permanently sustain functional capacity across a wide range of thermal conditions is costly for eurytherms, diverting energy away from somatic growth and reproduction. ${ }^{48,57,59,70,71}$ Conversely, thermal specialists, such as tropical or polar stenotherms, maximize their fitness in a narrower thermal niche and minimize maintenance costs, but exhibit increased capacity for phenotypic plasticity and acclimation responses. ${ }^{59}$

Future studies should investigate whether thermal specialists may also have a greater potential for rapid adaptation to climate warming, making them less vulnerable than their thermal generalist counterparts. Indeed, a reduction of the acclimatization response seems to be involved in adapting to a range of climatic conditions (i.e., becoming a thermal generalist $\left.^{53,58,72,73}\right)$. Consequently, this may render thermal generalists more vulnerable to global warming, since they may lack the potential to accomplish further niche shift via either plastic responses or rapid evolution..$^{747}$ In specialists, because of the steeper slope of the metabolic curves, a small shift in thermal niche width produces a sizeable change in temperature-dependent metabolic costs (Fig. 3).

\section{Water loss in terrestrial insects: a trade-off between water conservation and gas exchange}

Water loss potentially provides a direct link between the thermal niche of a species and its respiratory physiology. Water loss or transpiration can occur via cuticular transpiration or via gas exchange (respiratory transpiration), although typically the majority occurs via the former (70-80\% of total water loss). Especially in terrestrial insects, breathing dry, warm air may challenge water balance, owing to their small surface area-to-volume ratio. Additionally, their low thermal inertia can lead to rapid increases in body 
Temperate-adapted species-EURYTHERMAL

The loss of functional capacity coincides with the upper limit of environmental temperature
Tropical-adapted species-STENOTHERMAL

The functional capacity is efficiently sustained beyond the range of environmental temperatures

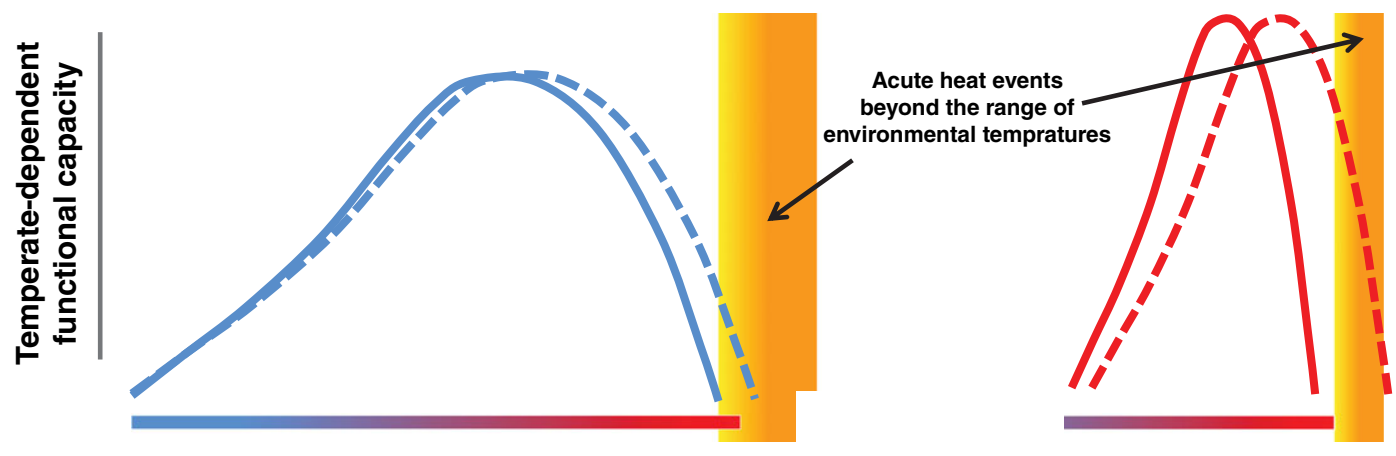

\section{Environmental temperature}

Figure 2. Conceptual model of different susceptibility to climate warming between temperate (eurythermal) and tropical (stenothermal) species. Temperature-sensitive species/populations (blue line) adapted to a high-latitude climate sustain temperature-dependent performances on a wide thermal niche but are more vulnerable to the effects of stochastic anomalous warming. Comparatively, tropical species/populations, particularly adapted to a more stable climate, may endure modest increases of environmental temperatures. Note that the anomalous heating events (orange areas) are less intense at tropical than at temperate regions, as predicted by the current projection on climate change. Modified from Ref. 68.

temperature, thereby increasing the water vapor saturation deficit (i.e., the difference between the actual water vapor pressure and the saturation vapor pressure). This means that insects and many other small ectotherms may desiccate faster and experience more rapid fluctuations in body temperature compared to larger organisms, all else being equal (e.g., morphology, color). Water loss may be tightly coupled with metabolic rate through the respiratory contribution of water lost from the mainly airfilled tracheae. Both water-loss rates and metabolic rate generally increase exponentially with increasing

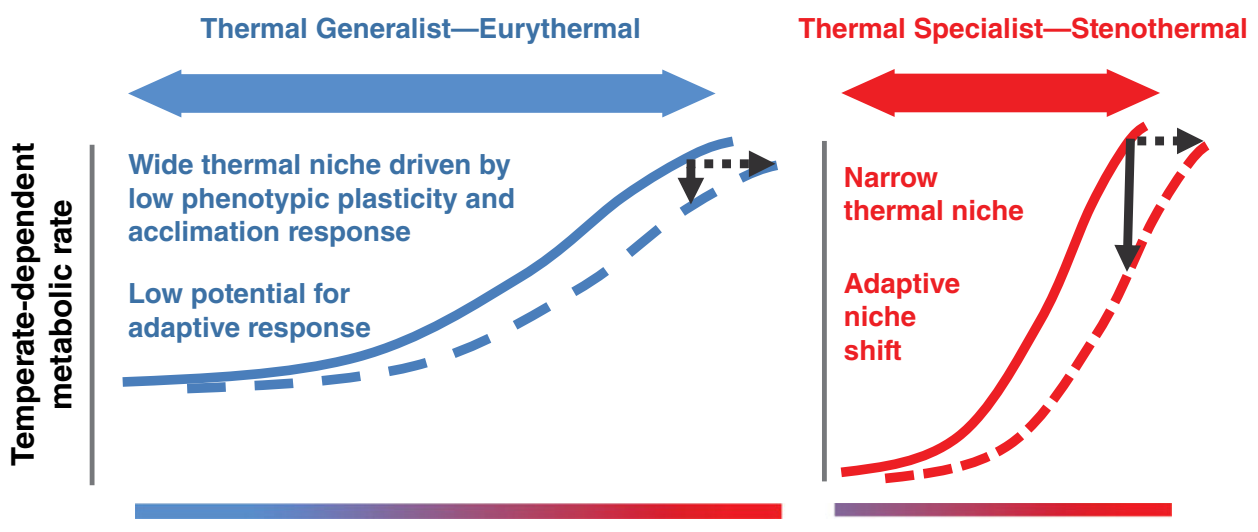

Environmental temperature

Figure 3. Projections of different adaptive strategies to climate warming between thermal generalist and specialist species. Thermal generalist species are able to sustain metabolic rate across a wide thermal niche thanks to compensatory mechanisms of respiratory functions. However, this pronounced phenotypic plasticity can reduce the potential for adaptive responses to anomalous climate warming. In contrast, thermal specialist species, confined in a narrower thermal niche, show limited acclimation response and may be more susceptible to niche shifts driven by evolutionary adaptations. Note that, thanks to the different skewness of the metabolic rate curves, minor niche shift (dotted black arrows) would be mirrored by different absolute variation of metabolic rate between generalist and specialist species (solid black arrows). 
temperature (but see the section on temperatureinsensitive metabolism, below). Hence, elevated, sublethal temperature conditions may increase the rate at which body water becomes depleted. It can therefore be argued that high temperature-related physiological limits are also imposed through indirect effects on metabolic rate and concomitant water loss in terrestrial animals, especially if there is limited access to water and food resources during the stress period. ${ }^{120}$

As the drying power of the air increases (i.e., increasing saturation deficit), it is expected that the contribution of respiratory transpiration to total water loss will increase, necessitating biochemical, physiological, morphological, and behavioral adaptations to tolerate or resist desiccation. Indeed, several early studies revealed distinct relationships between metabolic rate and water-loss rate that differed between insect species (mainly beetles) from mesic and xeric environments, ${ }^{76,77}$ with xeric species showing lower water-loss rate at a given metabolic rate and showing a clear relationship between water loss and metabolic rate. ${ }^{77}$ In addition, a generally positive association exists between gas-uptake rate and water-loss rate in resting insects. $^{78,79}$

Nevertheless, linking patterns in water-loss rates to fitness of insects under different thermal regimes is complicated. For example, even detailed assessments of differences in water-loss rates and survival times, considering the influence of saturation deficit separately from that of temperature and humidity, did not result in any clear patterns of association with habitat moisture availability in four species of Glossina flies, ${ }^{80}$ despite the fact that broad correlations exist among the water-loss rates and environments of these species, also after accounting for phylogenetic relatedness. ${ }^{81}$ Further confounding the intuitive importance of body condition via resource-depletion and hydration status is that, when insects are starved or desiccated for several days before heat- (or cold-) tolerance estimation, or exposed to combinations of both stressors, their lethal thermal limits remain remarkably unaffected. ${ }^{82,83}$

Given the mixed results obtained for simple direct tests of these predictions, two broad possibilities arise. Either these relationships between thermal niches and respiratory water loss genuinely do not exist, or methodological issues have con- founded the outcomes to some extent (e.g., mismatch between acute acclimation and evolutionary time scales, differences in respirometry approaches employed, and variation induced by methodology for estimating thermal limits). While some consider that a link between water-loss rates and thermal niches requires respiratory water loss to constitute a large part of total water loss, this is not necessarily the case. Indeed, even a small reduction in respiratory water loss may still introduce a fitness advantage within a natural population. ${ }^{84}$ In addition, and perhaps more importantly, if water balance is challenged under warm, dry conditions, one would reason that insects attempt to minimize respiratory water loss in response to such harsh environmental conditions. Insects may modulate respiratory transpiration through variation in pattern (e.g., discontinuous gas exchange (DGE)), and minimizing water loss has been proposed as one of the main drivers for DGE patterns. For example, Nauphoeta cinerea cockroaches that show DGE have lower water-loss rates and live longer than individuals not showing DGE. ${ }^{85}$ One way to effect a change is through modification of the spiracular (or tracheal) cross-sectional area (e.g., via spiracular behavior, shifts in gas-exchange pattern, or modulation in gross metabolic rate), thus influencing the basic Fick equation for gas transfer. ${ }^{86}$ Precisely how respiratory water loss might be modulated by metabolic rate is unclear and controversial. ${ }^{83,87}$ Regardless, insect eggs typically exert rapid control over the oxygenwater trade-off, ${ }^{88}$ indicating a selective advantage thereof.

One potential solution to the conflicting evidence on modulation of respiratory transpiration obtained to date is that trade-offs and hierarchies of control (or fundamental system constraints) prevent all species from responding equally or in a similar manner to the same external stressors. There are multiple solutions to the problem of maintaining water balance, with insects relying on different solutions. This point was already well appreciated in Hadley's scheme, ${ }^{89}$ which comprised five adaptive types: behavioral avoidance; enhanced water conservation; dehydration tolerance; high fluid turnover; and water sorption dependence. The capacity to mount a specific response may vary among species or taxonomic groups, each within its own constraints set by the environmental conditions or body plan, with some constraints taking 


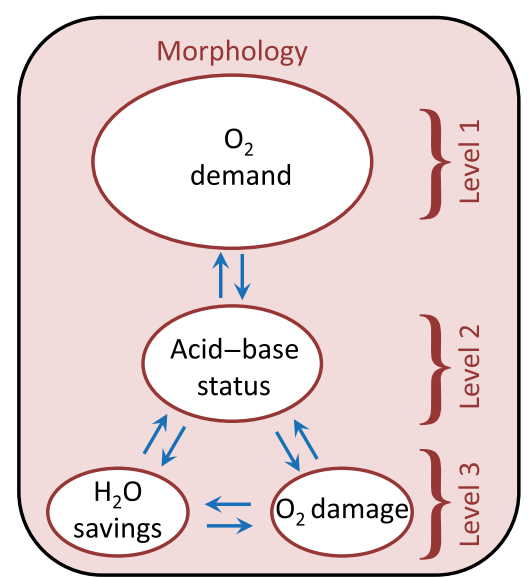

Figure 4. Schematic hierarchy of abiotic stressors influencing whole-animal gas-exchange responses. Different levels indicate stressors prioritized by the insect in decreasing order of importance, with level 1 indicating the stressor that is of highest priority to the animal; level 3 indicates stressors that can only be regulated once priorities from levels 1 and 2 have been satisfied. Arrows represent the interactions between the various stressors. All physiological regulation takes place within the context of the species' morphology. Adapted from Ref. 90.

precedence over others to give rise to a hierarchy of constraints. ${ }^{123}$

In conclusion, hierarchies of control and tradeoffs among competing demands within the same system to achieve different objectives need to be considered carefully in tests of adaptive hypotheses for respiratory modulation (Fig. 4). This therefore suggests that an integrative, systems biology view is required to address the problem of gas, water, and energy flux through an organism's respiratory system to better understand thermal adaptations.

The respiratory system of insects, and typically all air-breathing animals, first prioritizes sustained cellular aerobic metabolism by optimizing oxygen delivery, probably owing to the far more ATP-rich aerobic pathways of glycolysis. Second, the system attempts to maintain a homeostatic set point for $\mathrm{pH}$, which can also be regulated by respiratory excretion of $\mathrm{CO}_{2}$. Only if both of these two higher-level priorities (oxygen and $\mathrm{pH}$ balance) have been satisfied and have some innate flexibility (e.g., $\mathrm{pH}$ buffer capacity) or tolerance to perturbation can the system be employed to attempt to minimize oxidative damage and reduce respiratory water loss. ${ }^{90}$ The capacity for any further modulation will depend to some extent on metabolic demand (e.g., activity state), morphological structure (e.g., internal air stores), and acid-base buffer capacity. It is thus reasonable to assert that respiratory physiology can inform our understanding of thermal niches and, hence, climate change impacts of insects. However, further work is needed to understand the generalities and exceptions from an evolutionary physiological perspective for improved robustness of predicting any potential impacts.

\section{Temperature-insensitive metabolism (TIM) as a strategy to cope with fluctuating thermal environments}

The increase of metabolism with temperature may be a boon or a burden. Indeed, more-active animals can acquire resources more quickly, have higher dispersal rates, and can better escape from predators, and temperature usually increases growth rates and assimilation efficiency. ${ }^{91}$ However, the energetic costs required to drive elevated performance can become a burden, especially if resources are scarce or their uptake is intrinsically constrained by capacity limitations on activity and feeding. The adaptive value of metabolic depression, the lowering of whole-organism aerobic or total metabolism (including anaerobiosis) as a means to conserve energy when resting, has long been recognized during diapause (developmental cessation) and quiescence/estivation (response to environmental change) in ectotherm animals, as well as during torpor and hibernation (associated with a shift in the thermoregulatory set point) in endotherms (summarized in Ref. 92). Yet despite this, current perspectives for animal fitness give little consideration to energy conservation associated with depressed resting metabolism, largely equating energy surplus with net energy intake through activity and feeding. ${ }^{93}$ Energy-conserving metabolic depression at benign temperatures represents a different physiological response than the stress-related depressed aerobic performance. ${ }^{59}$ The latter is associated with insufficient capacity for oxygen delivery at an organism's thermal tolerance limit and ultimately causes mortality.

Thus, depressed metabolism may be adaptive or nonadaptive. In animals with adaptive metabolic depression, metabolism can be thermally insensitive (TIM) over a broad temperature range, and becomes thermally dependent again at higher temperatures (Fig. 5A). This recovery at high 
A

Temperature-independent depression

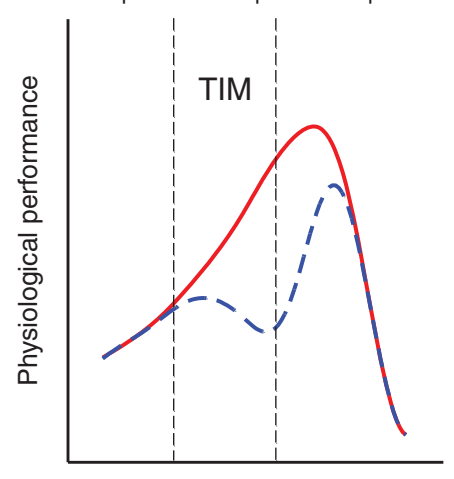

B

Temperature-independent depression

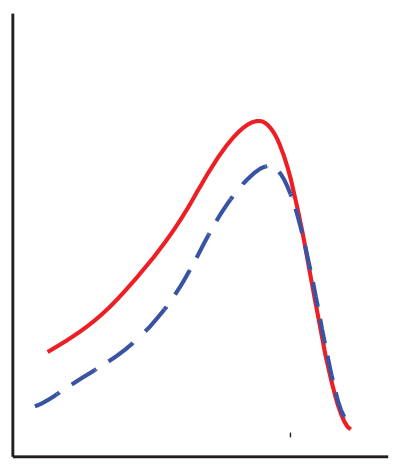

C

Stress-related depression

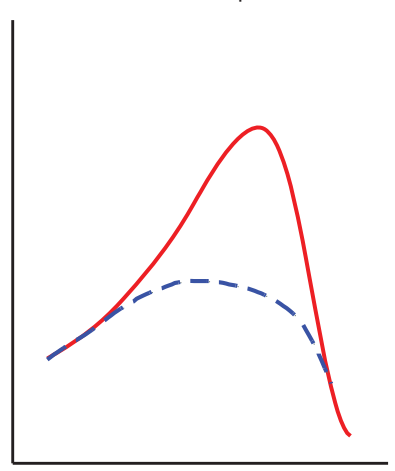

Temperature

Figure 5. Resting physiological (metabolic) performance curves (red lines) and patterns of depressed metabolism (blue dashed lines). (A) Adaptive temperature-insensitive metabolism (TIM) appropriate to intertidal animals experiencing widely varying temperatures, showing recovery of thermal dependence as heating increases, presumably to support a heat shock response. (B) Adaptive metabolic depression across the whole temperature range, appropriate to animals that behaviorally select stable estivating thermal environments. (C) Nonadaptive, capacity-limited temperature-insensitive metabolism constrained by resource or respiratory-related stress.

temperatures in animals whose heat-coma temperature has been exceeded presumably generates the energy to support a heat shock response. ${ }^{96}$ Alternatively, nonadaptive metabolic depression can be seen as unavoidable, being forced upon an organism from lack of food or oxygen, whereby metabolism shows no recovery of temperature dependence (Fig. 5C). In addition, metabolism may be depressed but still thermally dependent across the whole temperature range (Fig. 5B), which likely occurs in stable, stenothermal environments. This type of metabolic depression is exhibited by terrestrial gastropods that estivate in behaviorally selected stenothermal habitats. ${ }^{94}$ In contrast, TIM seems especially well developed in the uppermost intertidal gastropods that experience strong temperature fluctuations on a daily basis, elevated by direct solar exposure, while experiencing food and energy intake constraints. ${ }^{95,96}$ In the eulittoral fringe zone, the time period and thermal range for foraging is limited by infrequent wetting, and by snails rapidly becoming inactive under rising aerial temperatures-well below their thermal tolerance limits-to reduce desiccation risk. ${ }^{96}$

Energy gain during feeding is already constrained in gastropods by their generally low scope for activity and costly mode of locomotion. ${ }^{97}$ Whereas many ectotherms, especially highly active ones, are more or less capable of thermally adapting performance breadth for locomotion to match the environmental thermal range (for above-zero temperatures), ${ }^{48}$ similar adaptive selection of locomotor performance in gastropods is limited by their narrowed thermal breadth for activity and reduced scope of locomotion. TIM is not confined to intertidal gastropods, but has rather evolved independently along several lineages occurring in discrete marine ecological situations, including rocky shore and sandy beach cnidarians (Actinia), mollusks (Bullia, Patella, Littorina, Echinolittorina, Mytilus, Crassostrea) and crustaceans (Carcinus, Sphaeroma). ${ }^{98-105}$ The common factor in these cases is the combination of unpredictable food intake and environmental thermal variability. TIM is comparatively poorly known in terrestrial ectotherms. Although studies are increasingly revealing exceptions to the "universal temperaturedependence hypothesis" for metabolism of these animals, ${ }^{1}$ their behavioral capabilities to buffer environmental temperature change ${ }^{106}$ should lessen the advantage of evolving TIM.

Animals that exhibit TIM show very different thermal sensitivities of respiration and metabolism when comparing active animals to resting animals (Fig. 5). Yet the limits of their fundamental thermal niche (acute thermal tolerance) are ultimately 
set by aerobic physiological capabilities (Fig. 5). However, survivorship and fitness in thermally heterogeneous environments relate largely to energetic surplus, which in these animals becomes compromised when temperatures rise above the thermal limit for resting energy-conserving TIM, when an energetically costly heat shock response is induced ${ }^{96}$ (Fig. 5A). This contrasts with the situation for most ectotherms, in which energetics are compromised by environmental warming above the optimum temperature, when performance becomes suboptimal. ${ }^{63}$ Such contrasting scenarios suggest a need to better understand the links between respiration, metabolism, fitness, and thermal niche of ectotherms from a perspective considering all mechanisms that enhance energy surplus, not just temperature-related active metabolic performance above some standard resting level. The metabolic rate in resting animals does not necessarily conform to rate-temperature principles of physics as advocated by Brown and colleagues ${ }^{107}$ and may be adaptive. Including resting metabolism and the consequences of depression of resting metabolism for the energy balance of an animal will provide a better understanding of its thermal niche, especially for those animals living in potentially energy-sapping hot environments, while being constrained in their activity and feeding. ${ }^{98,99}$

\section{Understanding field observations from physiological principles}

Field observations provide invaluable data when comparing changes in field abundance, performance, or mortality to the tolerance of species to temperature extremes. Different ontogenetic stages may differ in their vulnerability to extreme field temperatures. ${ }^{121}$ For example, in Atlantic cod, adult spawners and the earliest life stages may be vulnerable to warming winters. ${ }^{108}$ Thermal stress may also constrain specific life phases and associated activities such as the spawning migration of salmon ${ }^{61}$ or reproductive success in littoral crabs. ${ }^{109}$ Observations of populations at the edge of their thermal range are most informative, revealing effects of extreme temperatures that may occur during either summer or winter, for example the decline of eelpout abundance following summer extremes in the Wadden Sea, where it reaches its southern distribution limit. ${ }^{60}$ On a larger scale, there is additional evidence that species are shifting their biogeographic distribution in response to climate warming, which according to the recent International Panel on Climate Change fifth assessment report ${ }^{110}$ is considered the most important driver of ecosystem changes. Ocean warming has resulted in distribution shifts of a wide range of marine species, and these have already led to changes in ecosystem composition and function. ${ }^{110,111}$ In order to understand these distributional shifts as well as the differences in response between species, one needs to focus on thermal performance limitations. These performance limitations will set in before death (i.e., sublethal limits), and the resulting loss in performance will have ecological impacts that result either directly from population dynamics or indirectly from species interactions. While it is well known that thermal constraints exist at lower levels of biological organization (individual protein molecules, organelles like mitochondria, individual cells), the OCLTT concept integrates these into the context of the whole organism. ${ }^{51,59}$ According to the OCLTT concept, sublethal performance limitations arise through a mismatch between the capacity and cost of the oxygen-supply system in relation to tissue oxygen demand. These are constraints experienced at the highest level of functional complexity by affecting more body compartments than other processes ${ }^{39,112}$ (Fig. 6). Evidence that constraints act at this highest level of complexity is provided by the observation that molecular and organellar functions display wider thermal tolerance ranges when they are isolated from the organism. ${ }^{113}$ However, within the context of the organism, these molecular and organellar functions are affected by the higher-order thermal constraints of the whole organism (e.g., by hypoxemia and associated oxidative stress (cf. Ref. 114)). This means that individual molecular or organellar functions in vivo may become limited earlier than when extracted from the organism context. Characterizing the role of oxygen supply and demand at the whole-organism level and aerobic metabolism at the mitochondrial level as mechanisms underlying thermal limitation thus requires acknowledging their interaction and feedback loops across different levels of organization in whole organisms. 


\section{Thermal windows across organisms}

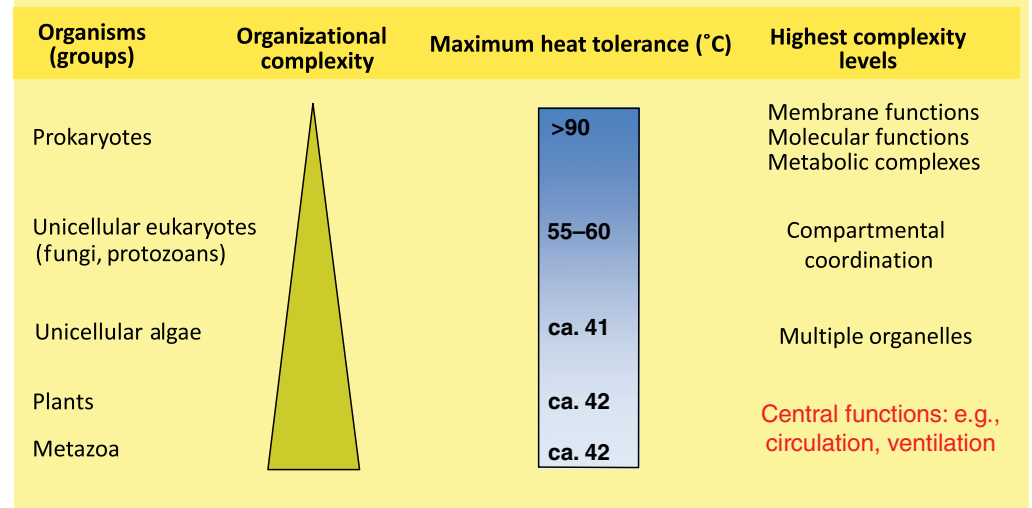

Figure 6. The rise in organizational complexity between prokaryotes, unicellular eukaryotes, and metazoans (cf. Ref. 112) correlates with a drop in the upper limits of thermal tolerance. Thus, limits to whole-organism thermal tolerance seem to be set at the highest complexity level (the level involving the largest number of compartments), which in animals comprises cells and organs involved in oxygen supply and demand. Modified from Ref. 39.

\section{Conclusion: can respiratory physiology predict thermal niches?}

Organisms can survive, grow, and reproduce if the energy they obtain is sufficient to cover all metabolic costs associated with their everyday life plus the amount required to cope with environmental variability. For water-breathers, the oxygen required to generate energy is more likely to become limiting than for air-breathers, and hence air-breathers are better equipped to meet increased or varying oxygen demand under cyclic (daily or seasonal) and anomalous warming (e.g., unpredictable heat event and climate change). In water, there is evidence that oxygen is directly involved in setting thermal limits, thus forging a direct link between respiration physiology and thermal niches. On land, oxygen is more plentiful and other stressors, notably related to water conservation and energy allocation, may come into play. In both media, the minimization of energy losses and water losses is achieved through physiological adaptations such as the evolution of lower metabolic rates or discontinuous breathing. These adaptations link respiration and warming, with higher temperatures resulting in increased gas exchange to maintain aerobic capacity, enhanced respiratory transpiration affecting the water balance, or more energy being diverted to maintenance affecting the energy balance. Thus, they exemplify various ways in which respiration can limit the thermal niche dimensions of a species.
A central point in our review is that elevated metabolic rates can be viewed both as a boon and a burden. When metabolism represents the energetic maintenance costs (standard metabolic rate), it becomes clear that increased metabolism diverts energy away from growth and reproduction, thus reducing the fitness of an organism. Alternatively, metabolism can represent the flow of energy (aerobic scope), reflecting the capacity of an organism to be active, grow, and reproduce. Following this view, increased metabolism would also increase the fitness of an organism. It therefore matters how thermal responses in metabolism are quantified, whether something is constraining metabolism (e.g., insufficient oxygen delivery) as well as the context and time scale at which thermal responses in metabolism take place (diurnal scale, acclimation response, or rapid evolution). Therefore, a perspective on thermal niches from respiration physiology needs to be widened beyond measuring oxygen consumption rates and aerobic scope. Thermal sensitivity of metabolism and the capacity for acclimation or the lack thereof are important for the understanding of thermal specialization ${ }^{66}$ as well as the ability of an animal to regulate its oxygen consumption. ${ }^{122}$ In addition, water balance may be critical for terrestrial air-breathing ectotherms, while thermal constraints on food acquisition may have driven the evolution of TIM in intertidal animals in a manner that does not fit with current textbook theory. 
Clearly, different metabolic challenges occur depending on the respiratory medium and the thermal regime (low versus high, stable versus fluctuating), but also on the evolutionary adaptations of a species (e.g., gas-exchange mechanism, mode of locomotion, and thermal window for energy generation). Each of the arrays of adaptations that have evolved involves different ways to deal with the various problems raised by the environment, such as the need to restrict water loss or the evolution of temperature-insensitive metabolism to save energy across part of the thermal window. Through the adoption of a hierarchical framework that considers the urgency by which different requirements need to be met (oxygen uptake, $\mathrm{CO}_{2}$ excretion, water homeostasis, etc.), one can make sense of the-sometimes-contrasting experimental results found. More generally, an integrative, multivariate view incorporating respiratory challenges, thermal responses, and energetic consequences is likely to provide insight that would be impossible to achieve if each of these traits were considered in isolation, and may be helpful both to improve our understanding of how thermal niches have evolved and to project the consequences of climate change for organisms. Such an integrative view of climate impacts across levels of biological organization needs to be followed through in experimental work and should be included in future modeling efforts for projections of ecosystem change. Specifically, future research addressing the following questions will help improve our understanding of how thermal niches have evolved:

1. How taxonomically and ecologically widespread is TIM as a means to maintain positive energy budgets and is it always associated with rapid temperature fluctuations?

2. Does the scaling exponent of metabolic rate differ with temperature, and do these effects of temperature differ with respiratory medium (water or air)?

3. Is metabolic scaling causally linked to differences in growth trajectories?

4. Do differences in oxyregulatory ability among species predict the degree to which heattolerance limits are set by oxygen?

5. Under which conditions (e.g., time scale, severity of stress) is the thermal niche associated with respiratory water loss, and does this explain evolutionary patterns in respiratory water loss across taxa inhabiting contrasting environments?

6. Do polar stenotherms, tropical stenotherms, and temperate eurytherms differ consistently in thermal sensitivity of performance, acclimation capacities, and evolutionary potential?

\section{Acknowledgments}

This paper stems from the contributions by the authors to the symposium "Respiratory adaptations across thermal clines," presented at the 3rd International Congress of Respiratory Science, July 6-10, 2014, at Bad Honnef, Germany. Financial support for this symposium was kindly provided by Pyroscience GmbH, Germany. WCEPV gratefully acknowledges financial support from a Marie Curie FP7 Integration Grant within the 7th European Union Framework Programme (FP7-PEOPLE2012-CIG proposal No. 334048)

\section{Conflicts of interest}

The authors declare no conflicts of interest.

\section{References}

1. Dell, A.I., S. Pawar \& V.M. Savage. 2011. Systematic variation in the temperature dependence of physiological and ecological traits. Proc. Natl. Acad. Sci. U. S. A. 108: 1059110596.

2. Rubner, M. 1883. Über den Einfluss der Körpergrösse auf Stoff- und Kraftwechsel. Z. Biol.-Munich. 19: 536-562.

3. Krogh, A. 1916. Respiratory Exchange of Animals and Man. London: Longmans, Green and Co.

4. Thompson, D.W. 1917. On Growth and Form. Cambridge: Cambridge University Press.

5. Reynolds, P.S. 1997. Phylogenetic analysis of surface areas of mammals. J. Mammal. 78: 859-868.

6. Schmidt-Nielsen, K. 1984. Scaling: Why is Animal Size so Important? Cambridge: Cambridge University Press.

7. Glazier, D.S. 2010. A unifying explanation for diverse metabolic scaling in animals and plants. Biol. Rev. 85: 111138.

8. White, C.R. \& M.R. Kearney. 2014. Metabolic scaling in animals: methods, empirical results, and theoretical explanations. Compr. Physiol. 4: 231-256.

9. von Bertalanffy, L. 1957. Quantitative laws in metabolism and growth. Q. Rev. Biol. 32: 217-231.

10. Hou, C., K.M. Bolt \& A. Bergman. 2011. A general model for ontogenetic growth under food restriction. Proc. R. Soc. B 278: 2881-2890.

11. Glazier, D.S. 2005. Beyond the '3/4-power law': variation in the intra- and interspecific scaling of metabolic rate in animals. Biol. Rev. 80: 1-52.

12. White, C.R., P.B. Frappell \& S.L. Chown. 2012. An information-theoretic approach to evaluating the size and 
temperature dependence of metabolic rate. Proc. R. Soc. B 279: 3616-3621.

13. Farrell-Gray, C.C. \& N.J. Gotelli. 2005. Allometric exponents support a 3/4-power scaling law. Ecology 86: 20832087.

14. White, C.R., P. Cassey \& T.M. Blackburn. 2007. Allometric exponents do not support a universal metabolic allometry. Ecology 88: 315-323.

15. Robinson, W.R., R.H. Peters \& J. Zimmermann. 1983. The effects of body size and temperature on metabolic rate of organisms. Can. J. Zool. 61: 281-288.

16. Hayssen, V. \& R.C. Lacy. 1985. Basal metabolic rates in mammals: taxonomic differences in the allometry of BMR and body mass. Comp. Biochem. A Physiol. 81: 741754 .

17. Bennett, P.M. \& P.H. Harvey. 1987. Active and resting metabolism in birds: allometry, phylogeny and ecology. J. Zool. 213: 327-363.

18. Kolokotrones, T. et al. 2010. Curvature in metabolic scaling. Nature 464: 753-756.

19. White, C.R. \& M.R. Kearney. 2013. Determinants of interspecific variation in basal metabolic rate. J. Comp. Physiol. B. 183: 1-26.

20. Glazier, D.S. 2006. The 3/4-power law is not universal: evolution of isometric, ontogenetic metabolic scaling in pelagic animals. Bioscience 56: 325-332.

21. Glazier, D.S et al. 2011. Ecological effects on metabolic scaling: amphipod responses to fish predators in freshwater springs. Ecol. Monogr. 81: 599-618.

22. Finkel, Z.V., A.J. Irwin \& O. Schofield. 2004. Resource limitation alters the $3 / 4$ size scaling of metabolic rates in phytoplankton. Mar. Ecol.-Prog. Ser. 273: 269-279.

23. Jeyasingh, P.D. 2007. Plasticity in metabolic allometry: the role of dietary stoichiometry. Ecol. Lett. 10: 282-289.

24. Hayes, M.B et al. 2015. High temperature slows down growth in tobacco hornworms (Manduca sexta larvae) under food restriction. Insect Sci. 22: 424-430

25. Marsden, I.D., R.C. Newell \& M. Ahsanullah. 1973. The effect of starvation on the metabolism of the shore crab, Carcinus maenas. Comp. Biochem. Physiol. A Physiol. 45: 195-213.

26. Lane, J.M. \& J.M. Lawrence. 1979. The effect of size, temperature, oxygen level and nutritional condition on oxygen uptake in the sand dollar, Mellita quinquiesperforata (Leske). Biol. Bull. 157: 275-287.

27. Yang, T.H. \& G.N. Somero. 1993. Effects of feeding and food deprivation on oxygen consumption, muscle protein concentration and activities of energy metabolism enzymes in muscle and brain of shallow-living (Scorpaena guttata) and deep-living (Sebastolobus alascanus) scorpaenid fishes. J.Exp. Biol. 181: 213-232.

28. Rosenfeld, J. et al. 2015. Relationship between growth and standard metabolic rate: measurement artefacts and implications for habitat use and life-history adaptation in salmonids. J. Anim. Ecol. 84: 4-20.

29. Hou, C. et al. 2008. Energy uptake and allocation during ontogeny. Science 322: 736-739.

30. Killen, S.S., D. Atkinson \& D.S. Glazier. 2010. The intraspecific scaling of metabolic rate with body mass in fishes depends on lifestyle and temperature. Ecol. Lett. 13: 184193.

31. Verberk, W.C.E.P. \& D. Atkinson. 2013. Why polar gigantism and Palaeozoic gigantism are not equivalent: effects of oxygen and temperature on the body size of ectotherms. Funct. Ecol. 27: 1275-1285.

32. Ivleva, I.V. 1980. The dependence of crustacean respiration rate on body mass and habitat temperature. Int. Rev. Hydrobiol. 65: 1-47.

33. Hsia, C.C.W. et al. 2013. Evolution of air breathing: oxygen homeostasis and the transitions from water to land and sky. Compr. Physiol. 3: 849-915.

34. Hetz, S.K. \& T.J. Bradley. 2005. Insects breathe discontinuously to avoid oxygen toxicity. Nature 433: 516-519.

35. Weibel, E.R., C.R. Taylor \& H. Hoppeler. 1991. The concept of symmorphosis: a testable hypothesis of structurefunction relationship. Proc. Natl. Acad. Sci. U. S. A. 88: 10357-10361.

36. Gillooly, J.F. et al. 2001. Effects of size and temperature on metabolic rate. Science 293: 2248-2251.

37. Dejours, P. 1981. Principles of Comparative Respiratory Physiology. Amsterdam: Elsevier.

38. Verberk, W.C.E.P. et al. 2011. Oxygen supply in aquatic ectotherms: partial pressure and solubility together explain biodiversity and size patterns. Ecology 92: 1565-1572.

39. Pörtner, H.-O. 2002. Climate variations and the physiological basis of temperature dependent biogeography: systemic to molecular hierarchy of thermal tolerance in animals. Comp. Biochem. Physiol. A Mol. Integr. Physiol. 132: 739761.

40. Verberk, W.C.E.P., U. Sommer, R.L. Davidson \& M.R. Viant. 2013. Anaerobic metabolism at thermal extremes: a metabolomic test of the oxygen limitation hypothesis in an aquatic insect. Integr. Comp. Biol. 53: 609-619.

41. Woods, H.A. 1999. Egg-mass size and cell size: effects of temperature on oxygen distribution. Integr. Comp. Biol. 39: 244-252.

42. Hoefnagel, K.N. \& W.C.E.P. Verberk. 2015. Is the temperature-size rule mediated by oxygen in aquatic ectotherms? J. Therm. Biol. DOI: 10.1016/ j.jtherbio.2014.12.003.

43. Horne, C.R., A.G. Hirst \& D. Atkinson. 2015. Temperaturesize responses match latitudinal-size clines in arthropods, revealing critical differences between aquatic and terrestrial species. Ecol. Lett. 18: 327-335.

44. Verberk, W.C.E.P. \& D.T. Bilton. 2013. Regulatory ability in aquatic insects dictates their vulnerability to global warming. Biol. Lett. 9: 20130473.

45. Giomi, F. et al. 2014. Improved heat tolerance in air drives the recurrent evolution of air-breathing. Proc. R. Soc. B281: 20132927.

46. Ern, R. et al. 2014. Oxygen delivery does not limit thermal tolerance in a tropical eurythermal crustacean. J. Exp. Biol. 217: 809-814.

47. Giomi, F. \& H.-O. Pörtner. 2013. A role for haemolymph oxygen capacity in heat tolerance of eurythermal crabs. Front. Physiol. 4: 110.

48. Angilletta, M.J. 2009. Thermal Adaptation: A Theoretical and Empirical Synthesis. Oxford University Press. 
49. Burggren, W.W. 2014. Epigenetics as a source of variation in comparative animal physiology-or-Lamarck is lookin' pretty good these days. J. Exp. Biol. 217: 682689.

50. Hoffmann, A.A., S.L. Chown \& S. Clusella-Trullas. 2013. Upper thermal limits in terrestrial ectotherms: how constrained are they? Funct. Ecol. 27: 934-949.

51. Pörtner, H.-O. 2010. Oxygen- and capacity-limitation of thermal tolerance: a matrix for integrating climate-related stressor effects in marine ecosystems. J. Exp. Biol. 213: 881893.

52. Somero, G., E. Dahlhoff \& J.J Linn. 2008. Stenotherms and eurytherms: mechanisms establishing thermal optima and tolerance ranges. Soc. Exp. Biol. Sem. Ser. 59: 53-78.

53. Hoffmann, A.A. 1990. Acclimation for desiccation resistance in Drosophila melanogaster and the association between acclimation responses and genetic variation. J. Insect Physiol. 36: 885-891

54. Schlichting, C.D. \& M. Pigliucci. 1998. Phenotypic Plasticity: A Reaction Norm Perspective. Sunderland: Sinauer Associates, Inc.

55. Pörtner, H.-O. et al. 2006. Trade-offs in thermal adaptation: the need for a molecular to ecological integration. Physiol. Biochem. Zool. 79: 295-313.

56. Somero, G.N. 2011. Comparative physiology: a "crystal ball" for predicting consequences of global change. Am. J. Physiol. Regul. Integr. Comp. Physiol. 301: R1-R14.

57. Clarke, A. 2003. Costs and consequences of evolutionary temperature adaptation. Trends Ecol. Evol. 18: 573-581.

58. Huey, R.B. \& D. Berrigan. 1996. "Testing evolutionary hypotheses of acclimation." In Animals and Temperature: Phenotypic and Evolutionary Adaptation. Vol. 59, 205-237. Cambridge: Cambridge University Press.

59. Pörtner, H.-O. 2006. Climate-dependent evolution of Antarctic ectotherms: an integrative analysis. Deep Sea Res. II 53: 1071-1104.

60. Pörtner, H.-O. \& R. Knust. 2007. Climate change affects marine fishes though the oxygen limitation of thermal tolerance. Science 315: 95-97.

61. Eliason, E.J. et al. 2011. Differences in thermal tolerance among sockeye salmon populations. Science 332: 109-112.

62. Stillman, J.H. 2003. Acclimation capacity underlies susceptibility to climate change. Science 301: 65.

63. Dillon, M.E., G. Wang \& R.B. Huey. 2010. Global metabolic impacts of recent climate warming. Nature 467: 704-706.

64. Tewksbury, J.J., R.B. Huey \& C.A. Deutsch. 2008. Putting the heat on tropical animals. Science 320: 1296.

65. Huey, R.B. et al. 2012. Predicting organismal vulnerability to climate warming: roles of behaviour, physiology and adaptation. Philos. Trans. R. Soc. Lond. B. Biol Sci. 367: 1665-1679.

66. Seebacher, F., C.R. White \& C.E. Franklin. 2015. Physiological plasticity increases resilience of ectothermic animals to climate change. Nat. Clim. Change 5: 61-66.

67. Wittmann, A.C. et al. 2008. Indicators of oxygen- and capacity-limited thermal tolerance in the lugworm Arenicola marina. Clim. Res. 37: 227.

68. Fusi, M et al. 2014. Thermal specialization across large geographical scales predicts the resilience of mangrove crab populations to global warming. Oikos 124: 784-795.
69. Sokolova, I.M. et al. 2012. Energy homeostasis as an integrative tool for assessing limits of environmental stress tolerance in aquatic invertebrates. Mar. Environ. Res. 79: $1-15$.

70. Kooijman, S.A.L.M. 2010. Dynamic Energy Budget Theory for Metabolic Organisation. 3rd ed. Cambridge: Cambridge University Press

71. Paaijmans, K.P. et al. 2013. Temperature variation makes ectotherms more sensitive to climate change. Glob. Change Biol. 19: 2373-2380.

72. Van Buskirk, J. \& U.K. Steiner. 2009. The fitness costs of developmental canalization and plasticity. J. Evol. Biol. 22: 852-860.

73. Chown, S.L. et al. 2010. Adapting to climate change: a perspective from evolutionary physiology. Clim. Res. 43: 3 .

74. Hoffmann, A.A. \& C.M. Sgrò. 2011. Climate change and evolutionary adaptation. Nature 470: 479-485.

75. van Heerwaarden, B. \& C.M. Sgrò. 2014. Is adaptation to climate change really constrained in niche specialists. Proc. R. Soc. B 281: 20140396.

76. Zachariassen, K.E. et al. 1987. Transpiratory water loss and metabolism of beetles from arid areas in East Africa. Comp. Biochem. Physiol. A 86: 403-408.

77. Addo-Bediako, A., S.L. Chown \& K.J. Gaston. 2001. Revisiting water loss in insects: a large scale view. J. Insect Physiol. 47: $1377-1388$.

78. Woods, H.A. \& J.N. Smith. 2010. Universal model for water costs of gas exchange by animals and plants. Proc. Natl. Acad. Sci. U. S. A. 107: 8469-8474.

79. Groenewald, B. et al. 2013. Gas exchange patterns and water loss rates in the Table Mountain cockroach, Aptera fusca (Blattodea: Blaberidae). J. Exp. Biol. 216: 3844-3853.

80. Kleynhans, E. \& J.S. Terblanche. 2011. Complex interactions between temperature and relative humidity on water balance of adult tsetse (Glossinidae, Diptera): implications for climate change. Front. Physiol. 2: 74.

81. Kleynhans, E. \& J.S. Terblanche. 2009. The evolution of water balance physiology in Glossina (Diptera: Glossinidae): correlations with climate. Biol. Lett. 5: 93-96.

82. Overgaard, J., T.N. Kristensen \& J.G. Sørensen. 2012. Validity of thermal ramping assays used to assess thermal tolerance in arthropods. PLoS One 7: e32758.

83. Terblanche, J.S. et al. 2011. Ecologically relevant measures of tolerance to potentially lethal temperatures. J. Exp. Biol. 214: 3713-3725.

84. Chown, S.L. 2002. Respiratory water loss in insects. Comp. Biochem. Physiol. A 133: 791-804.

85. Schimpf, N.G., P.G.D. Matthews \& C.R. White. 2012. Cockroaches that exchange respiratory gases discontinuously survive food and water restriction. Evolution 66: 597-604.

86. Clusella-Trullas, S. \& S.L. Chown, 2008. Investigating onychophoran gas exchange and water balance as a means to inform current controversies in arthropod physiology. J. Exp. Biol. 211: 3139-3146.

87. Chown, S.L., J.G. Sørensen \& J.S. Terblanche. 2011. Water loss in insects: an environmental change perspective. J. Insect Physiol. 57: 1070-1084.

88. Woods, H.A. 2010. Water loss and gas exchange by eggs of Manduca sexta: trading off costs and benefits. J. Insect Physiol. 56: 480-487. 
89. Hadley, N.F. 1994. Water Relations of Terrestrial Arthropods. San Diego: Academic Press.

90. Groenewald, B., S.L. Chown \& J.S. Terblanche. 2014. A hierarchy of factors influence discontinuous gas exchange in the grasshopper Paracinema tricolor (Orthoptera: Acrididae). J. Exp. Biol. 217: 3407-3415.

91. Angilletta, M.J. \& A.E. Dunham. 2003. The TemperatureSize Rule in ectotherms: simple evolutionary explanations may not be general. Am. Nat. 162: 332-342.

92. Withers, P.C. \& C.E. Cooper. 2010. "Metabolic depression: a historical perspective.” In Aestivation: Molecular and Physiological Aspects. C.A. Navas \& J.E. Carvalho, Eds.: 1-23. Berlin: Springer-Verlag.

93. Gilchrist, G.W. 1995. Specialists and generalists in changing environments. I. Fitness landscapes of thermal sensitivity. Am. Nat. 146: 252-270.

94. Guppy, M. \& P.C. Withers. 1999. Metabolic depression in animals: physiological perspectives and biochemical generalizations. Biol. Rev. 74: 1-40.

95. Marshall, D.J. \& C.D. McQuaid. 2011. Warming reduces metabolic rate in marine snails: adaptation to fluctuating high temperatures challenges the metabolic theory of ecology. Proc. R. Soc. B 278: 281-288.

96. Marshall, D.J. et al. 2011. Thermal adaptation in the intertidal snail Echinolittorina malaccana contradicts current theory by revealing the crucial roles of resting metabolism. J. Exp. Biol. 214: 3649-3657.

97. Davies, M.S., \& J. Blackwell. 2007. Energy saving through trail following in a marine snail. Proc. R. Soc. B 274: 1233 1236.

98. Newell, R.C. \& G.M. Branch. 1980. The influence of temperature on the maintenance of metabolic energy balance in marine invertebrates. Adv. Mar. Biol. 17: 329-396.

99. Branch, G.M. et al. 1988. Temperature and food as factors influencing oxygen consumption of intertidal organisms, particularly limpets. Amer. Zool. 28: 137-146.

100. Brown, A.C. \& F.M. da Silva. 1979. The effect of temperature on oxygen consumption in Bullia digitalis Meuschen (Gastropoda, Nassaridae). Comp. Biochem. Physiol. A 62: 573-576.

101. Newell, R.C. 1969. Effect of fluctuations in temperature on metabolism of interidal animals. Amer. Zool. 9: 293-307.

102. Newell, R.C. \& B.L. Bayne. 1973. A review on temperature and metabolic acclimation in intertidal marine invertebrates. Neth. J. Sea Res. 7: 421-433.

103. Percy J.A. \& F.A. Aldrich. 1971. Metabolic rate independent of temperature in mollusc tissue. Nature 231: 393-394.

104. Griffiths, R.J. 1977. Thermal stress and the biology of Actinia equina L. (Anthozoa). J. Exp. Mar. Biol. Ecol. 27: 141-154.

105. Marsden, I.D. 1979. Seasonal oxygen consumption of the saltmarsh isopod Sphaeroma rugicauda. Mar. Biol. 51:329337.

106. Kearney, M.R., R. Shine \& W.P. Porter. 2009. The potential for behavioral thermoregulation to buffer "cold-blooded" animals against climate warming. Proc. Natl. Acad. Sci. U. S. A. 106: 3835-3840.

107. Brown, J.H. et al. 2004. Toward a metabolic theory of ecology. Ecology 85: 1771-1789.
108. Perry, A.L., P.J. Low, J.R. Ellis \& J.D. Reynolds. 2005. Climate change and distribution shifts in marine fishes. Science 308: 1912-1915.

109. Bartolini, F. et al. 2013. Climate change reduces offspring fitness in littoral spawners: a study integrating organismic response and long-term time-series. Glob. Change Biol. 19: 373-386.

110. Poloczanska, E.S. et al. 2014. "Cross-chapter box on observed global responses of marine biogeography, abundance, and phenology to climate change." In Climate Change 2014. Impacts, Adaptation, and Vulnerability. Part A: Global and Sectoral Aspects. Contribution of Working Group II to the Fifth Assessment Report of the Intergovernmental Panel on Climate Change. C.B. Field et al., Eds.: 123-127. Cambridge and New York: Cambridge University Press.

111. Pörtner, H.-O. et al. 2014. “Ocean systems.” In Climate Change 2014. Impacts, Adaptation, and Vulnerability. Part A: Global and Sectoral Aspects. Contribution of Working Group II to the Fifth Assessment Report of the Intergovernmental Panel on Climate Change. C.B. Field et al., Eds. Cambridge and New York: Cambridge University Press.

112. Storch, D. et al. 2014. Climate sensitivity across marine domains of life: limits to evolutionary adaptation shape species interactions. Glob. Change Biol. 20: 3059-3067.

113. Pörtner, H.-O., L.S. Peck \& G.N. Somero. 2012. "Mechanisms defining thermal limits and adaptation in marine ectotherms: an integrative view." In Antarctic Ecosystems: An Extreme Environment in a Changing World, 1st ed. A.D. Rogers et al., Eds.: 360-396. Blackwell Publishing Ltd.

114. Kassahn, K. et al. 2009. Animal performance and stress: responses and tolerance limits at different levels of biological organisation. Biol. Rev. 84: 277-292.

115. Fridovich, I. 1977. Oxygen is toxic! Bioscience 27: 462-466.

116. Speakman, J.R. 2005. Body size, energy metabolism and lifespan. J. Exp. Biol. 208: 1717-1730.

117. Verberk, W.C.E.P. \& D.T. Bilton. 2011. Can oxygen set thermal limits in an insect and drive gigantism? PLoS One 6: e22610.

118. Gilchrist, G.W. 1995. Specialists and generalists in changing environments. I. Fitness landscapes of thermal sensitivity. Am. Nat. 146: 252-270.

119. Verberk, W.C.E.P. \& D.T. Bilton. 2015. Oxygen limited thermal tolerance is seen in a plastron breathing insect, and can be induced in a bimodal gas exchanger. J. Exp. Biol. 218: 2083-2088.

120. Kleynhans, E., D.E. Conlong \& J.S. Terblanche. 2014. Direct and indirect effects of developmental temperature on adult water balance physiology of Eldana saccharina (Lepidoptera: Pyralidae). J. Insect Physiol. 68: 69-75.

121. Kingsolver, J.G., H.A. Woods, L.B. Buckley, et al. 2011. Complex life cycles and the responses of insects to climate change. Integr. Comp. Biol. 51: 719-732.

122. Deutsch, C., A. Ferrel, B. Seibel, et al. 2015. Climate change tightens a metabolic constraint on marine habitats. Science 348: $1132-1135$.

123. Matthews, P.G.D. \& J.S Terblanche. 2015. Evolution of the Mechanisms Underlying Insect Respiratory Gas Exchange. Adv. Insect Physiol. http://dx.doi.org/ 10.1016/bs.aiip.2015.06.004. 